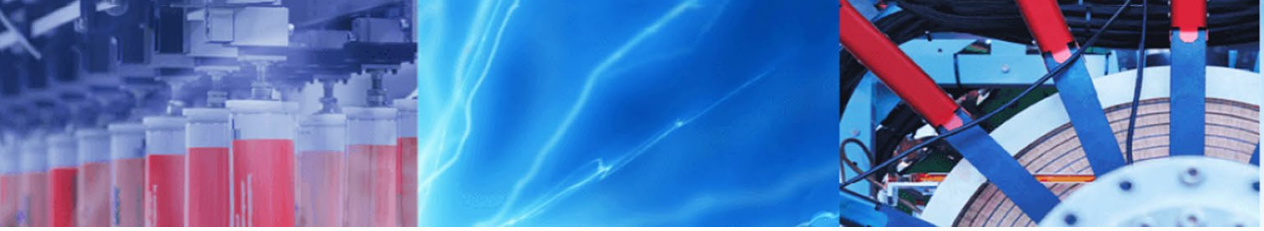

Research Article

\title{
Fog harvesting: combination and comparison of different methods to maximize the collection efficiency
}

\author{
Seyyedmajid Sharifvaghefi' ${ }^{1} \cdot$ Hanif Kazerooni $^{2}$
}

Received: 26 November 2020 / Accepted: 17 March 2021 / Published online: 31 March 2021

(C) The Author(s) 2021 OPEN

\begin{abstract}
Fog harvesting is an unconventional source of water that can be used in some regions with water scarcity to overcome water shortages. The most commonly used collectors are meshes which have intrinsic limitations, the most important of which are clogging and aerodynamic deviation of droplets around the wires. Here, three techniques are compared and combined to overcome these limitations, i.e., replacing the mesh with an array of vertical wires, addition of a hydrophobic layer to the wires, and forcing the ionized droplets to move toward the wires by applying an electric field. The combination of these techniques was found to result in higher fog harvesting efficiency compared to each individual method with the highest impact from the addition of the electric field. The combined methods lead to a 60 -fold increase in fog harvesting efficiency compared to meshes. The findings showed that when the fog droplets are forced in an electric field toward the wires, the shading coefficient for collectors can be increased to 1 from 0.55 (maximum for collectors without the electric field) without affecting the fog harvesting efficiency, allowing for lower construction cost of the collectors. Addition of the electric field showed two distinctive promotional effects. First, increasing the aerodynamic efficiency and second, reducing the size of droplets sliding down the wires by disturbing the three-phase contact line and reducing the contact angle hysteresis and the pinning force. Energy analysis shows that this technique can be 100 times more energy efficient compared to the conventional atmospheric water generators.
\end{abstract}

Keywords Fog harvesting · Efficiency $\cdot$ Contact angle $\cdot$ Vertical wires $\cdot$ Hydrophobic layer $\cdot$ Electric field

\section{Introduction}

Freshwater availability is now becoming an increasing problem worldwide. This problem is more likely to occur in arid and semi-arid regions with limited or no precipitation. In such areas, water scarcity is not only due to the scarcity of the whole freshwater mass, but also to the inadequate collection and conservation of water [1, 2].

There are many options available to improve the efficiency of water usage, but these may not be sufficient to meet human needs in many regions facing severe water shortages. Therefore, countries, regions, and communities facing water scarcity are increasingly looking at unconventional water resources to reduce the gap between water supply and demand. Among the different sources of unconventional water, fog harvesting has been suggested in recent decades as a potential way to collect water in arid and semi-arid regions. This method is mostly suitable for areas with high humidity and prone to fog formation. Many studies have focused on fog harvesting including the fabrication of biomimetic materials which has shown some success $[3,4]$.

Fog harvesters are most commonly made of mesh netting. These meshes are made in different sizes and placed perpendicular to the wind direction. The use of these meshes has shown success in many rural and coastal

Seyyedmajid Sharifvaghefi, s.sharifvaghefi@sharif.edu| ${ }^{2}$ Department of Chemical and Petroleum Engineering, Sharif University of Technology, Tehran, Iran. ${ }^{2}$ Department of Chemical Engineering, Amirkabir University of Technology (Tehran Polytechnic), Tehran, Iran. 
communities around the world $[5,6]$. The meshes can collect water droplets as they pass through their holes by wind. These droplets, which are small at first, coalesce and grow. They are then driven by gravity to a small channel at the bottom and then to a storage tank [7]. Although the meshes have achieved relatively good maturity due to its many years of development and cost-effectiveness, field studies has shown that they have low efficiency (around $1-2 \%)$ due to intrinsic limitations [8].

Researchers have studied the meshes to find the optimum configuration for fog harvesting by changing their geometry and wettability [9-12]. The main limitations related to the meshes are the shedding rate and aerodynamic deviation of fog droplets around the mesh wires. Low shedding rate of droplets can affect the efficiency through clogging of the openings of meshes. The main approach to maximize the shedding rate is to add coatings (mainly hydrophobic) to the meshes which can reduce the contact angle hysteresis of the droplets that attach to the surface, and therefore, facilitate their drainage [13, 14]. In a recent study, Shi et al. [15] have shown that removing the horizontal wires and only using the vertical array of wires on meshes (called harps), can be an effective way to increase the shedding rate of fog droplets to avoid clogging, and therefore, enhancing the efficiency of the collector.

To overcome the limitation related to the deviation of fog droplets around mesh wires, Damak and Varanasi [16] have adopted the electrostatic precipitation method, which is used to collect aerosol particles by applying an electrostatic field to the ionized particles. They showed that using this technique, the number of fog droplets that deposit on the wires increases dramatically. They used a sharp metal as an ion emitter to charge the fog droplets and collected the charged droplets on a grounded mesh. In the absence of the electric force, the droplets travelling toward the wires follow the air streamlines and with the effect of drag force, they move around the wires. In the presence of an electrostatic field, the electric forces are larger than the drag forces and the droplets follow the field lines and are collected on the wires.

Each of the mentioned methods has its own limitations when applied for fog harvesting. When using the array of wires instead of meshes, the fog droplets can pass around the wires, thereby reducing the efficiency. When using the electrostatic method to collect the fog droplets on a mesh, the low shedding rate and clogging of the mesh pores can lower the efficiency of the system. In this study, we have combined these methods to minimize the limitations with both the shedding rate and aerodynamic deviation of fog droplets around individual wires. In this way, we attempt to maximize the fog harvesting efficiency with our system. Combining these methods can overcome the limitations associated with each method when they are used separately. The results showed an overall $84 \%$ efficiency in the combined system which was higher than the efficiency of each method when used separately.

\section{Experimental methods}

The setup used for fog harvesting is shown in Fig. 1. The system includes a fog collector, an ultrasonic humidifier to generate fog, fog transfer lines, a high voltage generator with adjustable voltage (manufactured by Megavolts with a maximum voltage of $25 \mathrm{kV}$ ) and a resistance box (Megavolts) to change the output power of the system. Fog was formed using an ultrasonic humidifier with an output rate of $0.1 \mathrm{l} / \mathrm{h}$ and speed of $0.2 \mathrm{~m} / \mathrm{s}$ with an average fog droplet radius of $7 \mu \mathrm{m}$.

The water collection section was constructed as an array of vertical wires placed inside a clear plexiglass frame
Fig. 1 The experimental setup for fog harvesting with the main parts of the system shown

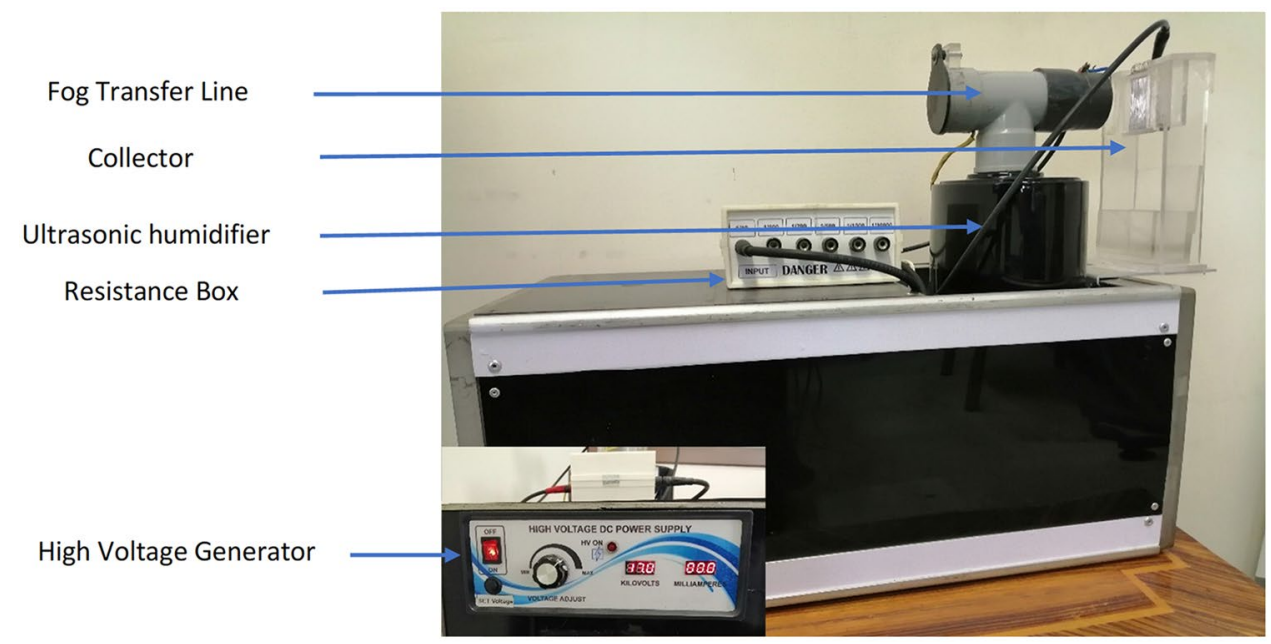


(Fig. 2). The wires were made of stainless steel. The overall dimensions of the plexiglass frame were about $4.5 \times 4 \mathrm{~cm}$. A reservoir for collecting water droplets was also placed underneath the wires. The wires were exposed to a fog stream a short distance from the humidifier outlet $(3 \mathrm{~cm})$.

The fog droplets were charged by placing an electrode (connected to the high voltage generator) at the fog outlet and connecting the other pole of the power supply to the collecting wires. The relative humidity of the outlet of humidifier was $100 \%$ which was measured by a digital hygrometer with an external probe (Benetech GM1361). The air velocity was measured using an anemometer (Benetech GM8903). At specific time intervals (30-60 min without charging the droplets and 15 min when droplets were charged) the weight of the reservoir used to collect water was measured by a scale to determine the amount of water obtained. The process was repeated five times for each test to determine the repeatability of the test. The (fog) harvesting efficiency is measured as:

Water generated from the humidifier - collected water Water generated from the humidifier

Three different diameters of wire were used in this study to build the collectors: $0.5,1$ and $1.5 \mathrm{~mm}$, which were named as W0.5-2, W1-2 and W1.5-2, respectively. If $D$ is the diameter of the wires and $P$ is the center distance between the wires, the $P / D$ ratio was equal to 2 for all the wires. This resulted in a shading coefficient (SC, i.e., the fraction of projected area covered by the wires) of 0.5 for all three wires. For the $0.5 \mathrm{~mm}$ diameter two more collectors were made with different spacing between the wires ( 2 and $3 \mathrm{~mm}$ ) resulting in a $P / D$ ratio of 4 and 6 , and were named W0.5-4 and W0.5-6. Although the aerodynamic efficiency $\left(\eta_{\mathrm{a}}\right)$ is shown to be at its maximum with $\mathrm{SC} \approx 0.55$, we were interested to examine the effect of $P / D$ ratio especially for the case with the electric force added. A mesh with wire diameter of $0.7 \mathrm{~mm}$ and $P / D$ ratio of 2.2 was also used for comparison and was named M0.7. Research has shown that these specifications are close to the optimum state for a mesh [8]. Figure 3 shows the fabricated collectors. The specifications of the collectors including the shading coefficient and the Stokes number are shown in Table 1.

To investigate the effect of the hydrophobic coating layers on the harvesting efficiency, a W0.5-2 collector was coated with the hydrophobic layer with a dip-coating method adopted from reference [17]. Briefly, a solution of isopropanol, dimethyldimethoxysilane and sulfuric acid with a weight ratio of 100/10/1 was prepared. The solution was stirred for about $30 \mathrm{~s}$ and allowed to stand for $30 \mathrm{~min}$ at room temperature before applied to the wires. After washing and cleaning the wires, they were dip-coated in the prepared solution for $5 \mathrm{~s}$ and dried at $40^{\circ} \mathrm{C}$ for $30 \mathrm{~min}$. The formation of the hydrophobic layer is based on sulfuric acid catalyzed hydrolysis and polymerization of dimethyldimethoxysilane. This collector was named W0.5-2H. The characteristics of the W0.5-2 wires and droplets formed on it in the presence of the electric field were studied and the results are shown under W0.5-2E collector.

\section{Results and discussion}

\subsection{Fog harvesting without the electric field}

Figure 4 shows the fog harvesting efficiency for each of the collectors. The highest fog harvesting efficiency was obtained with W0.5-2. By increasing the diameter of the wires, the harvesting efficiency decreased by $44 \%$ and $64 \%$ for $W 1-2$ and $W 1.5-2$, respectively. This trend was also shown by Shi et.al [15]. Increasing the gap between wires (increasing $P / D$ ratio) also reduced the harvesting efficiency by $27 \%$ and $48 \%$ for W0.5-4 and W0.5-6, respectively. The data also suggest that the
Fig. 2 The fog droplets' capture and collection section of the fog harvesting system

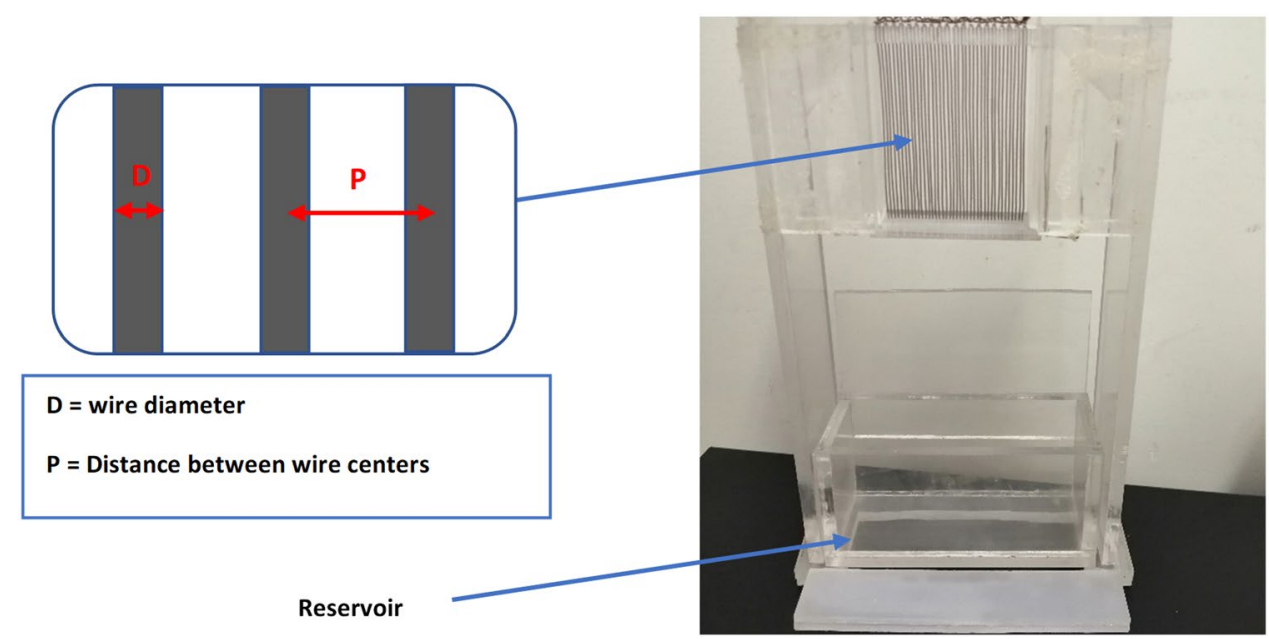

SN Applied Sciences a SPRINGER Nature journa 
Fig. 3 Different wire and mesh collectors used for fog harvesting
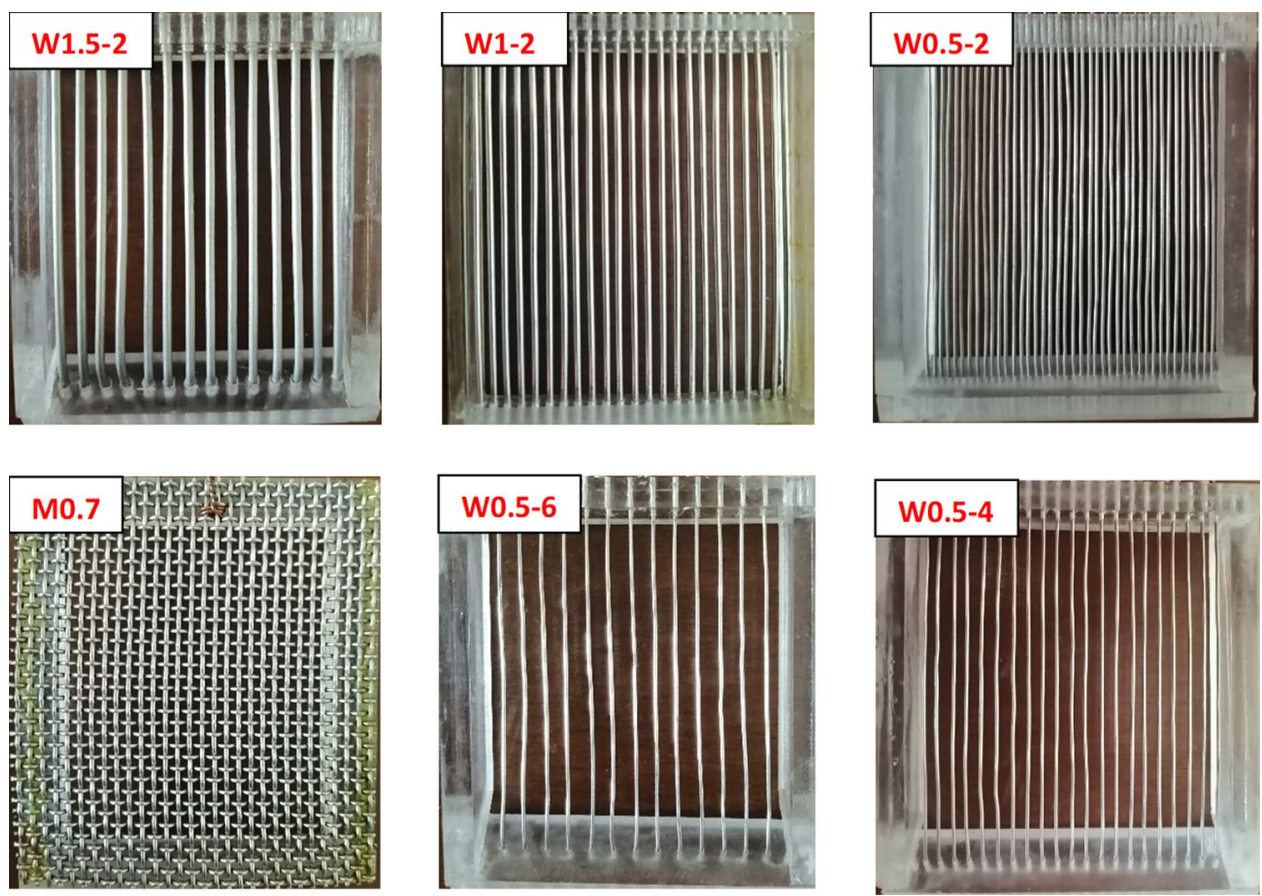

Table 1 Wire diameter $(D)$, ratio of the center-to-center distance between wires to the wire diameter $(P / D)$, shade coefficient $(S C)$, and Stokes number (St), receding and advancing contact angles $\left(\theta_{r}, \theta_{a}\right)$, contact angle hysteresis $\left(\theta_{\mathrm{h}}\right)$, and the empirical and theoretical critical volume of drops $\left(V_{c, \text { e }}, V_{c, t}\right)$ for each of the collectors

\begin{tabular}{|c|c|c|c|c|c|c|c|c|}
\hline Collector & $D(\mathrm{~mm})$ & $P / D$ & SC & St & $\theta_{\mathrm{r}}$ (deg) & $\theta_{\mathrm{a}}$ (deg) & $V_{c, e}(\mu \mathrm{L})$ & $V_{c, t}(\mu \mathrm{L})$ \\
\hline W0.5-2 & 0.5 & 2 & 0.5 & 0.48 & $35 \pm 3$ & $53 \pm 5$ & $1.7 \pm 0.8$ & $2.70 \pm 1.2$ \\
\hline W1-2 & 1 & 2 & 0.5 & 0.24 & $34 \pm 4$ & $54 \pm 2$ & $2.0 \pm 0.9$ & $3.00 \pm 0.8$ \\
\hline W1.5-2 & 2 & 2 & 0.5 & 0.16 & $34 \pm 5$ & $53 \pm 6$ & $2.5 \pm 0.5$ & $4.05 \pm 2.1$ \\
\hline W0.5-4 & 0.5 & 4 & 0.25 & 0.48 & $35 \pm 3$ & $53 \pm 2$ & $1.6 \pm 0.7$ & $1.35 \pm 0.6$ \\
\hline W0.5-6 & 0.5 & 6 & 0.17 & 0.48 & $35 \pm 3$ & $53 \pm 2$ & $1.6 \pm 0.5$ & $1.35 \pm 0.6$ \\
\hline W0.5-2H & 0.5 & 2 & 0.5 & 0.48 & $151 \pm 3$ & $154 \pm 5$ & $1.2 \pm 0.5$ & - \\
\hline M0.7 & 0.7 & 2.2 & 0.45 & 0.34 & $35 \pm 3$ & $52 \pm 5$ & $5.9 \pm 1.3$ & - \\
\hline W0.5-2E & 0.5 & 2 & 0.5 & 0.48 & $66 \pm 3$ & $72 \pm 4$ & $0.7 \pm 0.3$ & $0.61 \pm 0.4$ \\
\hline
\end{tabular}

effect of increasing the diameter of wires in reducing the fog harvesting efficiency is greater than increasing the distance between wires. Compared to the mesh collector, all wired collectors showed higher fog harvesting efficiencies. By comparison, the fog harvesting efficiency in the W0.5-2 collector was about 3 times higher than the mesh collector used, which is a significant increase. It is worth noting that the mesh used in our experiments, as mentioned earlier, has the optimum dimensions for fog harvesting.

In short, the collecting wires used here outperformed the mesh in fog harvesting, and as the diameter of the wires and the distance between them decreases, the fog harvesting efficiency is increased. In other words, thinner and closer wires can more effectively collect and shed droplets.
As mentioned before, the main problem with the mesh collectors is the clogging of their openings with the deposited droplets. The ability of the wires to prevent clogging is related to the reduction in pinning force, which allows easier shedding of the captured droplets down the wires. In case of mesh collectors, the horizontal wires prevent the smooth shedding of the droplets.

As shown in Fig. 4, with the addition of the hydrophobic layer to the mesh collector, the harvesting efficiency was increased by $62 \%$. This very well illustrates the reason for the presence of hydrophobic layers on the leaf surface of some plants. The hydrophobic layer was also added to the W0.5-2 collector to investigate its effect on the wire collectors. Compared to the untreated W0.5-2 collector, $29 \%$ increase was observed after the addition of the hydrophobic layer. The increase in fog harvesting efficiency is about 2 times lower 
Fig. 4 Fog harvesting efficiency for each of the collectors

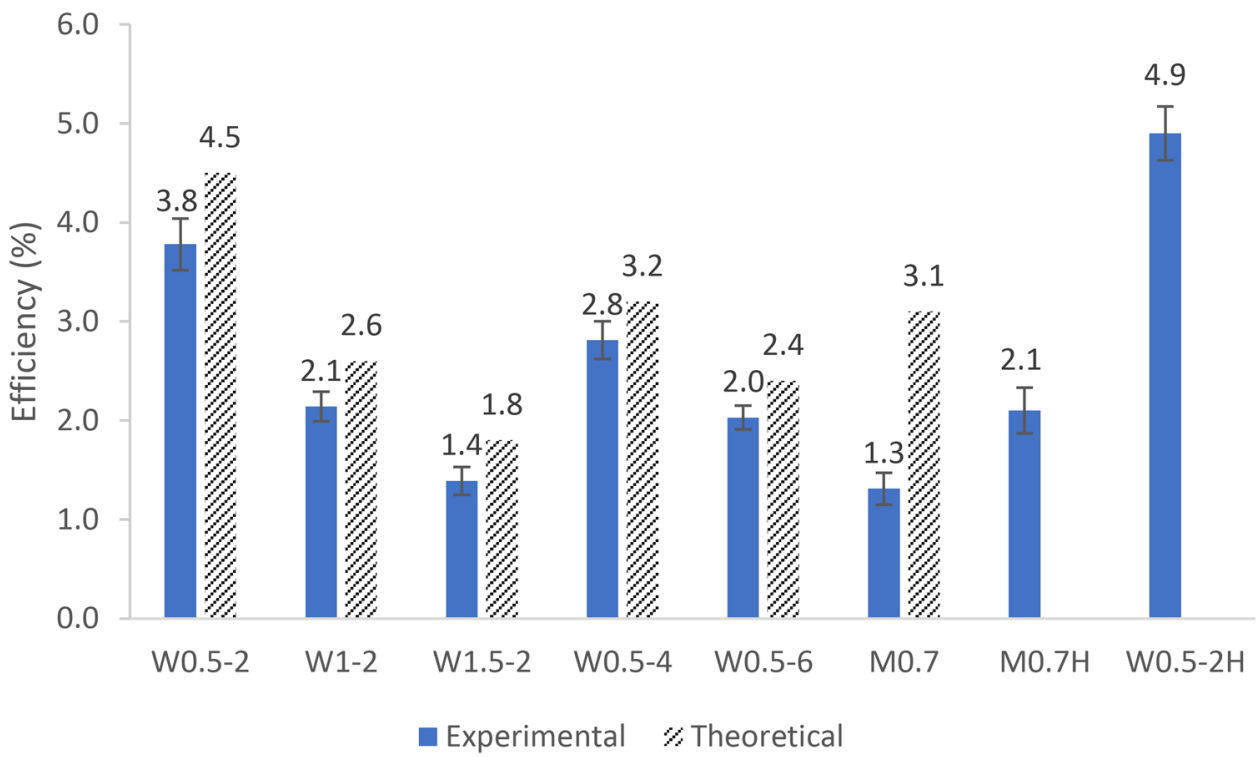

The above equation is valid for $\operatorname{Re}<400$ which is the case in this work. Deposition efficiency depends on the Stokes (St) number, which is the ratio of the droplet inertia to the drag force and shows the ability of the droplets to follow streamlines and is shown as [8]:

$\mathrm{St}=\frac{2 \rho_{\text {water }} \cdot U_{0} \cdot r_{\text {fog }}^{2}}{9 \mu_{\text {air }} \cdot R_{\text {wire }}}$

where $\rho_{\text {water }}$ is water density, $U_{0}$ is wind speed, $r_{\text {fog }}$ is the radius of water droplets in fog, $\mu_{\text {air }}$ is air viscosity, and $R_{\text {wire }}$ is the radius of wire. Larger values of St lead to more droplets on the wires of a collector, while smaller values lead to more droplets around the wires. The numerical values of St are listed in Table 1 for each wire and mesh collector. The deposition efficiency is a direct function of the Stokes number:

$\eta_{\mathrm{d}}=\frac{\mathrm{St}}{\mathrm{St}+\frac{\pi}{2}}$

We assigned the test conditions where $r_{\text {fog }}=7 \mu \mathrm{m}$ and $U_{0}=0.2 \mathrm{~m} / \mathrm{s}$ to find the values for Re, $C_{\mathrm{d}}, C_{0}, \eta_{\mathrm{a}}$, and $\eta_{\mathrm{d}}$ for different collectors, as shown in Table 2 . The theoretical fog harvesting efficiency using the calculated values for the tested collectors are shown in Fig. 4. The experimental efficiency found for the W0.5-2 collector was 3.8\%, which is slightly below the theoretical value of $4.6 \%$, indicating that experimental results reasonably follow the theoretical values. The difference between the theoretical and experimental results

where

$A_{\operatorname{Re}}=\left(4.6 \times 10^{-11}\right) \cdot \operatorname{Re}^{4}-\left(7.1 \times 10^{-8}\right) \cdot \operatorname{Re}^{3}+\left(3.8 \times 10^{-5}\right) \cdot \operatorname{Re}^{2}-8.7 \cdot \operatorname{Re}+1.78, \quad \operatorname{Re}=\rho_{\text {air }} v_{0} D / \mu_{\text {air }}$ 
Table 2 Values for $\mathrm{Re}, A_{\mathrm{Re}}$ $C_{0}, C_{d}$, and deposition and aerodynamic efficiencies for the tested collectors

\begin{tabular}{lrlllrl}
\hline Collector & \multicolumn{1}{l}{$\operatorname{Re}$} & $A_{\mathrm{Re}}$ & \multicolumn{1}{l}{$C_{0}$} & $C_{\mathrm{d}}$ & $\eta_{\mathrm{d}}(\%)$ & $\eta_{\mathrm{a}}(\%)$ \\
\hline W0.5-2 & 6.73 & 1.72 & $2.85 \mathrm{E}+00$ & 1.18 & 23.31 & 19.59 \\
W1-2 & 13.46 & 1.67 & $2.76 \mathrm{E}+00$ & 1.18 & 13.19 & 19.77 \\
W1.5-2 & 20.19 & 1.62 & $2.67 \mathrm{E}+00$ & 1.18 & 9.20 & 19.96 \\
W0.5-4 & 6.73 & 1.72 & $7.52 \mathrm{E}-01$ & 1.18 & 23.31 & 13.90 \\
W0.5-6 & 6.73 & 1.72 & $4.53 \mathrm{E}-01$ & 1.18 & 23.31 & 10.49 \\
M0.7 & 9.42 & 1.70 & $1.08 \mathrm{E}+01$ & 1.18 & 17.84 & 17.38 \\
\hline
\end{tabular}

is probably due to the fact that part of the generated fog condenses on the plexiglass frame. It should be noted that for the mesh collector used in our experiments, the clogging problems significantly impede the aerodynamics of the system which results in lower efficiency than the predictions. For example, for the M0.7 collector, the fog harvesting efficiency was only $1.3 \%$, which is far from the predicted value (3.1\%).

The effective shedding of droplets collected down the wires can be measured using the contact angle hysteresis model. This may help to better understand why reducing the diameter of wires increases the efficiency of water harvesting. As shown by previous reports [20,21], the pinning force is due to the formation of contact angle hysteresis and acts along the receding contact line. A drop on a wire can be in the barrel position or just on one side of the wire [22]. For the falling droplets, the first case (barrel state) was observed in W0.5-2 collector, while in other collectors only the second case was observed (Fig. 5).

The experimental critical volume of the sliding droplets can be approximated based on their shape as shown by Shi et al. [15]. The droplet volume sliding down one
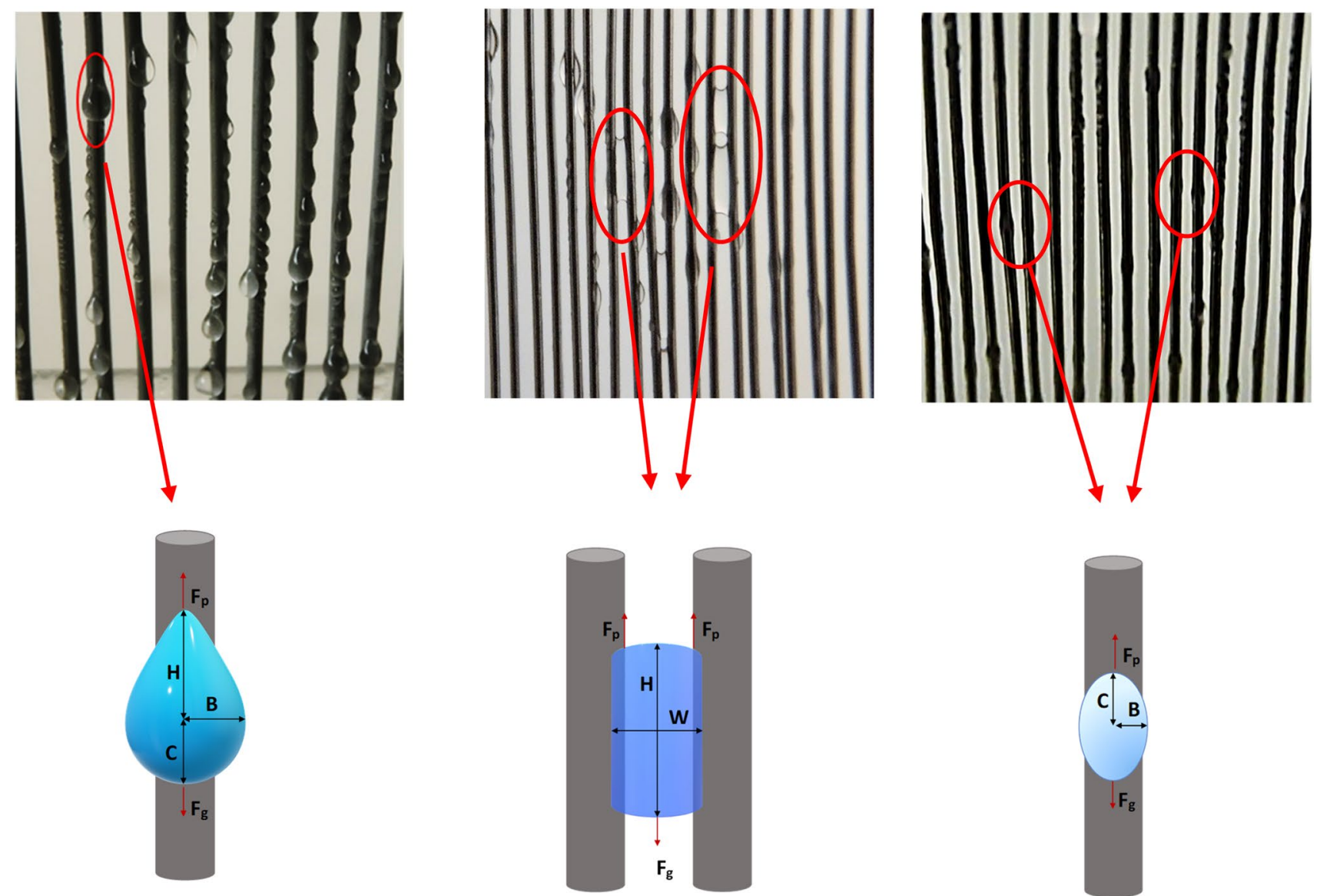

Fig. 5 The drop and barrel shape of the sliding droplets on the W1.5-2 (left), W0.5-2 (middle), and W0.5-2E (right) collectors and their corresponding illustrations

\section{SN Applied Sciences}


side of a vertical wire can be considered as the sum of a half cone and a quarter spheroid $\left(V=\frac{\pi B^{2} H}{6}+\frac{4 \pi B^{2} C}{12}\right)$ and the volume of the barrel can be approximated as a cuboid $(V=W H D)$. The volume of the drop sliding down the wires when the electric field is present can be approximated as a half spheroid $\left(V=\frac{4 \pi B^{2} C}{6}\right)$. Analyzing five different images of a drop starting to slip on a wire, the average $V_{\mathrm{c}, \mathrm{e}} \approx 2.5 \pm 0.5 \mu \mathrm{L}$ can be calculated for W1.5-2 collector by geometric simplifications. The experimental critical volume of the drops in barrel state was found by considering a cuboid shape for the drop and finding the dimensions of cuboid through five measurements. The empirical value of $V_{c, e}=1.7 \pm 0.8 \mu \mathrm{L}$ was obtained in this way for W0.5-2 collector (Table 1). The above results show that the critical droplet volume is proportional to the size of the wires. One can understand why thin wires cause better water sliding and thus prevent clogging.

Considering the length of receding contact line to be $\pi R_{\text {wire, }}$ the theoretical critical volume for droplets sliding down on one side of the wires can be calculated as [15]:

$\rho_{\text {water }} g V_{\mathrm{c}, \mathrm{t}} \approx \pi R_{\text {wire }} \sigma\left(\cos \theta_{\mathrm{r}}-\cos \theta_{\mathrm{a}}\right)$

where $\sigma$ is the surface tension and $g$ is gravity. Using the values in Table 1 for receding and advancing contact angle values, the theoretical critical volumes can be obtained as $3.00 \pm 0.6$ and $4.05 \pm 2.1 \mu \mathrm{L}$ for the W1-2 and W1.5-2 collectors, respectively. For the W0.5-2 collector, where the water slides down in between the wires in a barrel shape, the critical volume can be found as [15]:

$\rho_{\text {water }} g V_{\mathrm{c}, \mathrm{t}} \approx 2 \pi R_{\text {wire }} \sigma\left(\cos \theta_{\mathrm{r}}-\cos \theta_{\mathrm{a}}\right)$

The difference between the two equations is the presence of an extra 2 on the right-hand side of the second equation for the dual receding contact lines of the barrel shape water sliding down in between the wires. Using this equation, the theoretical critical volume of the W0.5- 2 collector is $2.7 \pm 1.2 \mu \mathrm{L}$. The results show that the theoretical critical volumes follow the same trend as the experimental ones. The difference between the theoretical and experimental critical volumes can be due to the simplified equations used for calculating the theoretical critical volumes which are based on Furmidge model.

As shown in Table 1, with the addition of a hydrophobic layer on the wires, the critical volume of droplets decreases as they slide down the wires. This reduction in volume makes the droplets shed more easily and faster compared to the untreated surface. In other words, the shelf life of the droplets on the surface is reduced, which ultimately leads to an increase in the efficiency of fog harvesting. This reduction in critical volume is due to the drop in pin force on the wire surface.

\subsection{Fog harvesting with the help of an electric field}

As mentioned, the main mechanisms that limit the efficiency of the conventional fog collectors are the shedding rate and the aerodynamic deviation of the fog droplets. Improving the shedding rate was discussed in the previous section and overcoming the second limitation (deviation of droplets around wires of the collector) will be discussed here. The ability of the droplets to follow the streamlines is determined by the Stokes number. At low Stokes numbers, the droplets follow the flow paths closely, and small portion of droplets are collected. At high Stokes numbers, drag forces have no effect on the paths, and the droplets move toward the wires along their paths and collide with them. However, as shown in Table 1, the stokes number increases as the diameter of wires is reduced. Therefore, large Stokes number requires very fine wires that are difficult to construct and lack structural integrity. Hence, low deposition efficiency remains an important challenge in fog collection.

The solution suggested by Damak and Varanasi [16] is used in this section to overcome the aerodynamic limitation of the streamline deviation around the wires which is to introduce an additional electrical force that overcomes the aerodynamic drag force and directs the fog droplets toward the wires. Their approach was inspired by the principles of electrostatic adsorbents to increase fog collection $[23,24]$.

The mechanism of action of the addition of this force is schematically illustrated in Fig. 6. By connecting the ion emitter and the collecting wires to the power supply poles, an electric field is created between them. The droplets are ionized by the ion emitter and the applied electric field directs the ionized droplets toward the wires. The distance between the ion emitter and the collector can be divided into three regions, i.e., around the emitter, in-between, and close to the collector. The electric field lines move from the emitter toward the wires of collector and are strongest close to the emitter and weakest around the collector. The particle charging begins around the emitter. When the applied voltage increases, it reaches a certain value when corona discharge is produced. Free electrons are created at this voltage which are repulsed by the negative electric field toward the collector. With the electric force, the electrons accelerate and gain enough energy to ionize the air atoms (by liberating electrons) when they collide with them. All of this happens close to the emitter. A chain reaction occurs at this stage creating more and more electrons and ions. The created ions are attracted back toward the emitter and can collide with the air atoms around them, creating additional electrons.

Entering the in-between electrode area, the electrons are still repulsed by the emitter but the effect of the 
High volatge generator

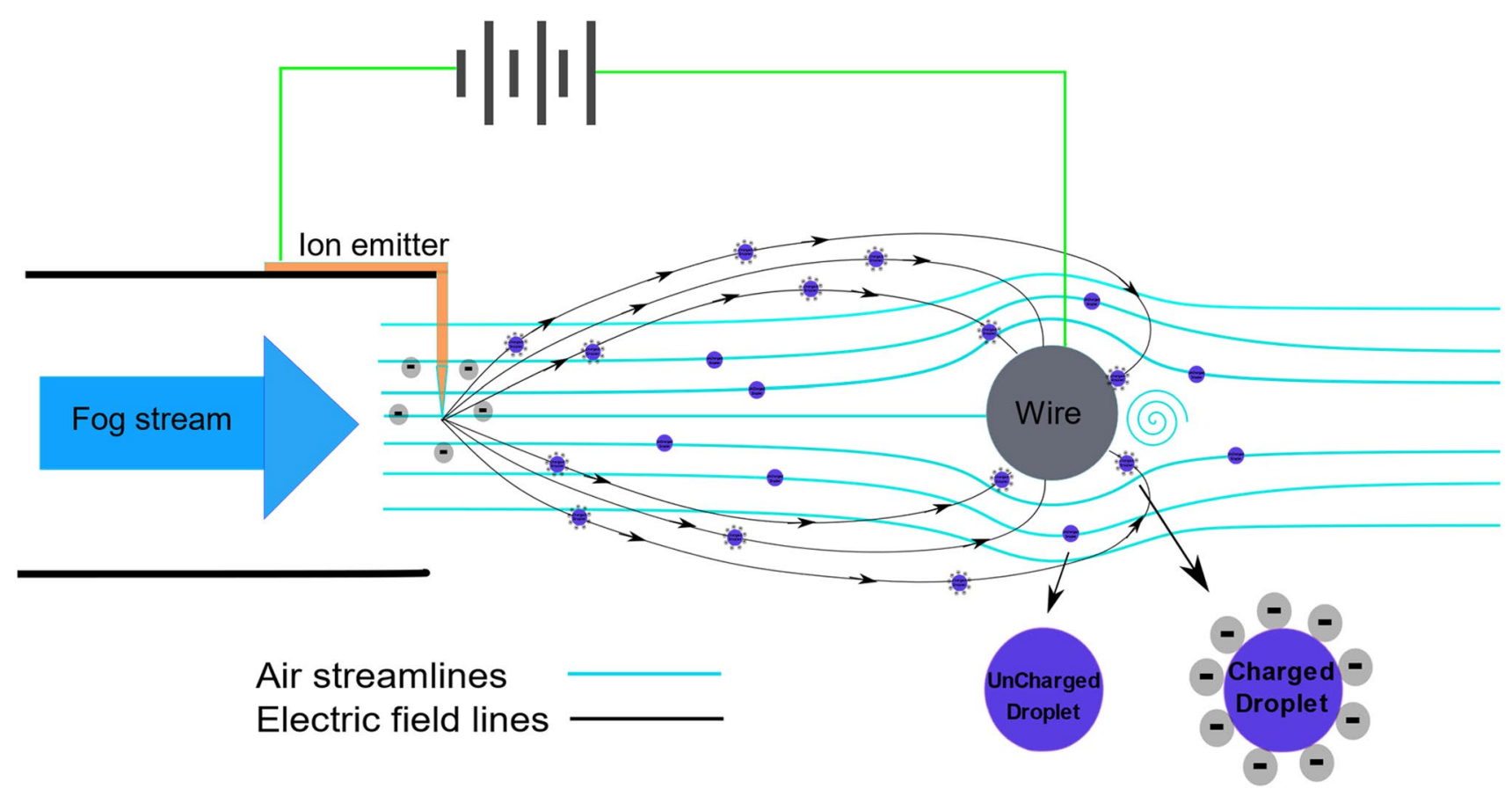

Fig. 6 A schematic of the air streamlines and electric field lines for the fog collecting system

electric field on the electrons is weaker. When electrons collide with the air atoms in this region, they are captured by the atoms and the electron charge is transferred to them. This creates negatively charged air ions. Since these ions are negatively charged, they move away from the emitter and toward the collector. This puts them in the same path as the fog droplets. Therefore, these negatively charged ions play the main role in ionizing and capturing of the fog droplets [25-27]. The ionization of droplets allows them to collect on the wires and some droplets that are not initially directed toward the wires are also captured.

The results of the fog harvesting efficiency in the presence of electric field in terms of $V^{2}$ for different wire collectors are shown in Fig. 7. The results indicate the extremely high efficiency of the system, which shows a 53-fold increase compared to fog harvesting without the use of electric field for the mesh collector. Here the wires of different diameters are shown in separate figures to better analyze them. Figure 7 a shows that increasing the diameter of the wires does not have much effect on the efficiency of fog harvesting, but the vertical wires are about $19 \%$ more efficient than the mesh in the maximum applied voltage. Increasing the distance between the wires (Fig. 7b), at lower voltages, allows for more fog droplets to pass through. However, at higher voltages, the stronger electric field can overcome the distance between the wires and more fog droplets are harvested. This is very interesting as the optimum $P / D$ ratio for fog harvesting without using an electric field is 2 which allows for the maximum aerodynamic efficiency [19]. The results show that when using the electric field, this general rule is no longer valid and with $P / D$ ratio of 4 , the same fog harvesting efficiency can be achieved. From an economical point of view, this allows the number of wires used to be reduced to half without sacrificing the efficiency which can lower the costs of the unit. Increasing the $P / D$ ratio to 6 resulted in lower fog harvesting efficiency indicating that the electric field cannot overcome the drag force with the increased distance. The results also show that, unlike before (without using electric current), the hydrophobic layer has little effect ( $3 \%$ increase) on increasing fog capturing efficiency. This indicates that in the case of using electric current, previous restrictions on droplets rolling off the wires have been largely eliminated.

As can be seen in Fig. 7, using the electric field, the fog harvesting efficiency increases linearly with $V^{2}$ as the output voltage increases at lower voltages. At a given voltage, the fog harvesting efficiency reaches its maximum and then there is no change in the fog harvesting efficiency as the voltage increases.

To better understand the changes in the efficiency versus the voltage, a dimensionless number $\left(k_{\mathrm{e}}\right)$ is introduced 

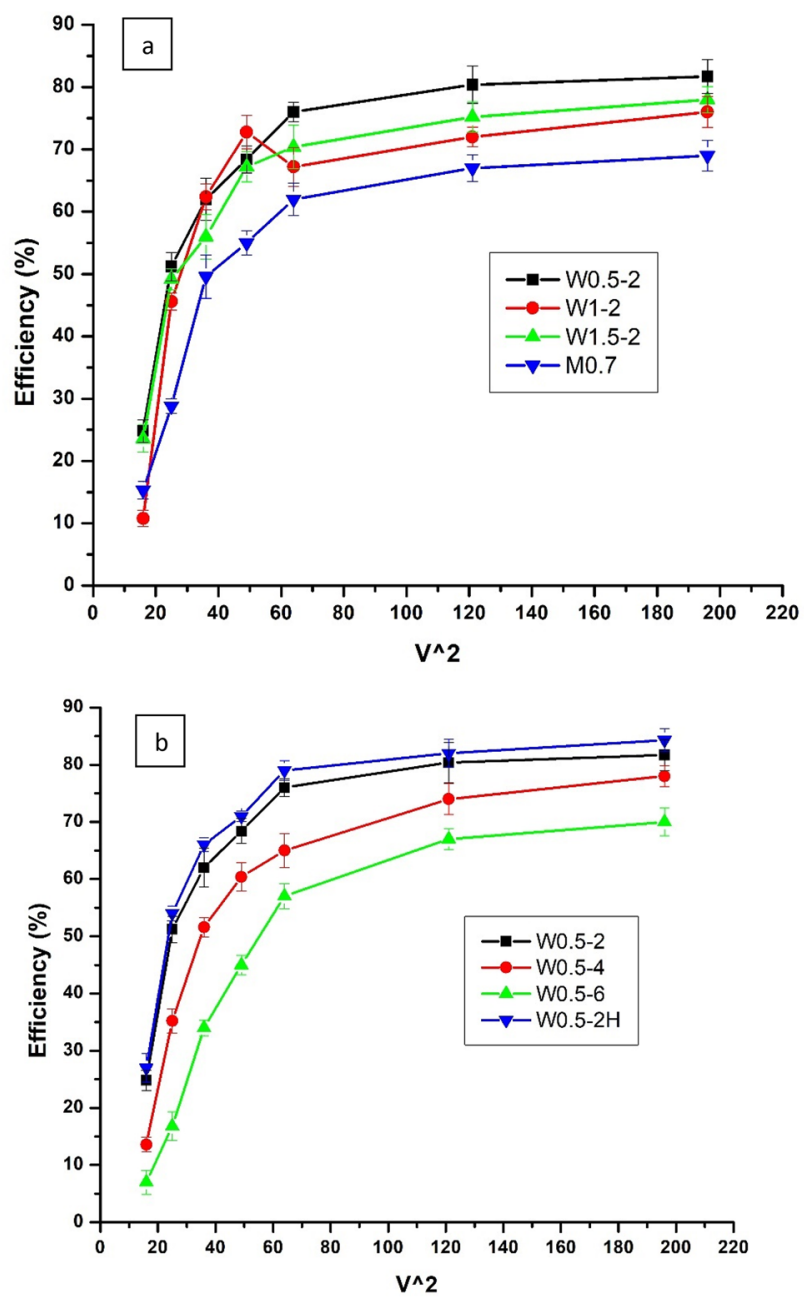

Fig. 7 Fog harvesting efficiency in the presence of electric field for different wire collectors

as, $K_{\mathrm{e}}=\frac{2 r_{\text {fog }} \cdot \varepsilon_{0} \cdot V^{2}}{\mu_{\text {air }} \cdot L \cdot R_{\text {wire }} \cdot U_{0}\left(1+U^{*}\right)}$ where $U^{*}=\frac{2 r_{\text {fog }} \cdot \varepsilon_{0} \cdot V^{2}}{\mu_{\text {air }} \cdot L^{2} \cdot U_{0}} \cdot K_{\mathrm{e}}$ is the ratio of the electric and viscous forces and is called the electric number and $U^{*}$ is a dimensionless number indicating the ratio between the speed added due to the electric field and the wind speed [16]. In these equations, $L$ is the distance between the ion emitter and the collector, $\varepsilon_{0}$ is the vacuum permittivity constant, and $U_{0}$ is the fog droplets initial speed. $K_{\mathrm{e}}$ is found to be an important factor in the process of fog harvesting and the output efficiency will be proportional to $K_{\mathrm{e}}\left(\eta \approx K_{\mathrm{e}}\right)$. For low $U^{*}$, the term $V^{2}$ in the denominator of $K_{\mathrm{e}}$ is negligible, thus $K_{\mathrm{e}}$ is linearly related to $V^{2}$ and hence, the output efficiency increases linearly with $V^{2}$. However, for high values of $U^{*}, U_{0}$ becomes negligible in the denominator and $K_{\mathrm{e}}$ tends toward a constant value $\left(L / R_{\text {wire }}\right)$. Increasing the voltage beyond this point does not lead to the collection of more droplets. This is called the saturation voltage. The $K_{\mathrm{e}}$ equation can be written as $K_{\mathrm{e}}=L / R_{\text {wire }}\left(U^{*}\right) /\left(1+U^{*}\right)$. Complete saturation occurs when $U^{*}>>1$, but its effects start to show when $U^{*}=1$. The saturation voltage can be seen in bending the lines and the efficiency becoming almost constant with increasing the voltage as shown in Fig. 7.

Analyzing the shape of falling droplets for W0.5-2E (in the presence of electric field), a clear shift was observed from barrel position (as was seen for W0.5-2 collector) to drop shape (being on just one side of the wire) as shown in Fig. 2. This was related to the reduction in volume of droplets sliding down the wires. Analyzing the volume of droplets sliding down the wires for W0.5-2E (in the presence of electric field), a significant reduction (59\%) was observed when compared to W0.5-2 (without the electric field). Moreover, the contact angle hysteresis of the falling drops also showed a three-fold decrease. The reduction in the volume of droplets can be attributed to the reduction in pinning force which is related to contact angle hysteresis as shown in the following equation [28]:

$f_{\mathrm{p}}=-\sigma w\left(\cos \theta_{\mathrm{r}}-\cos \theta_{\mathrm{a}}\right)$

where $f_{\mathrm{p}}$ is the pinning force, $\sigma$ is the surface tension and $w$ is the width of the droplet and the minus sign show the opposite direction of pinning force in regard to the moving direction of droplet. The equation shows that with the reduction in $\mathrm{CAH}$, the pinning force is decreases allowing for faster roll off of droplets collected on the wires. The reduction in $\mathrm{CAH}$ can be attributed to the presence of the electric field which perturbs the force balance at the three-phase contact line. This can lead to the depinning of the contact line from the surface and faster shedding of droplets which in turn can free more condensing areas on wires for droplet nucleation and growth. The same phenomenon is also observed in electrowetting resulting in similar changes to the $\mathrm{CAH}$ [29-31]. The electric field is therefore, has two distinctive advantages: first, increasing the aerodynamic efficiency of the system by directing the fog droplets toward wires and second, by reducing the pinning force and therefore, increasing the shedding rate.

\subsection{Investigation of energy consumption and comparison with atmospheric water generators}

We used a resistance box (Fig. 1) to control the output current and thus investigate the amount of energy required for fog harvesting with the help of an electric field. The output current of the power supply is about $1 \mathrm{~mA}$. With the help of the resistance box, this output can be reduced to $1 / 10,000$. Figure 8 shows the fog harvesting efficiency for collector W0.5-2 at different output currents. The results showed that the minimum output current to reach the 


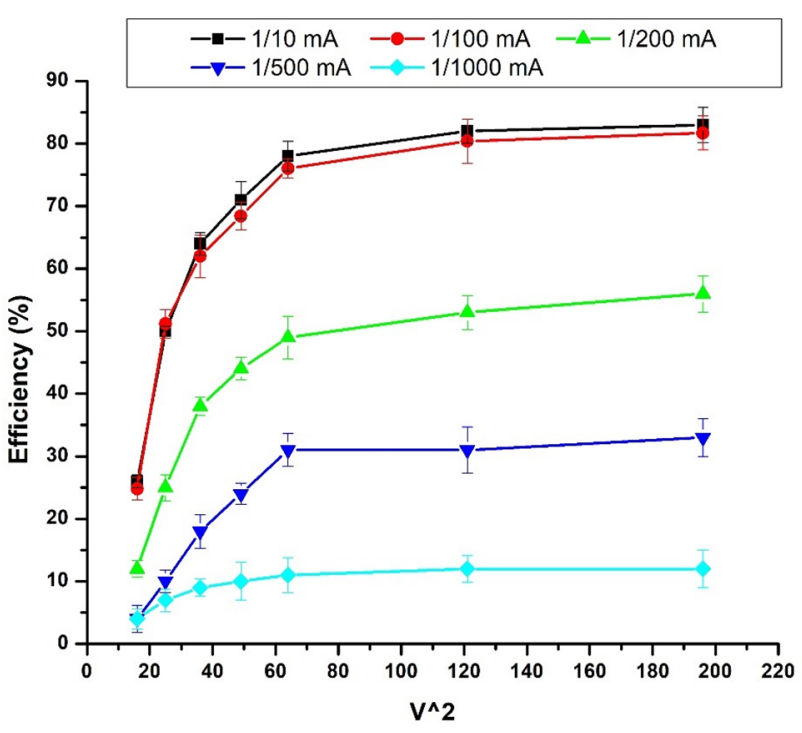

Fig. 8 Fog harvesting efficiency for W0.5-2 collector at different output currents

maximum efficiency was about $0.01 \mathrm{~mA}$. Lowering the current output from $0.01 \mathrm{~mA}$ resulted in lower maximum efficiency. Therefore, the tests in study were performed with $1 / 100$ of the current output from the power supply (about $0.01 \mathrm{~mA}$ ). Below the $0.01 \mathrm{~mA}$ output, the number of ions emitted is not sufficient to ionize the fog droplets which results in lower amount of water collected.

The results show that at high voltages $\left(V^{2}=196\right)$, using a current of $0.01 \mathrm{~mA}, 82 \%$ efficiency was achieved. The amount of power consumed in this case is about $0.1 \mathrm{~W}$. Therefore, for our collectors with dimensions of $4 \times 4.5 \mathrm{~cm}$, the power consumption per unit square meter is about $60 \mathrm{~W} / \mathrm{m}^{2}$. Considering the maximum water production in the system $(\sim 82 \mathrm{~g} / \mathrm{h})$, the amount of energy consumed is about $1.2 \mathrm{KWh} / \mathrm{m}^{3}$. The energy consumption in this method is therefore, about two order of magnitude lower than the amount of energy consumed in current atmospheric water generators $\left(\sim 200-500 \mathrm{KWh} / \mathrm{m}^{3}\right)$.

\section{Conclusion}

The fog harvesting was performed with an array of vertical wires which showed up to 3 times increase in efficiency compared to conventional nets. This increase was related to lack of restricting horizontal wires and therefore, the decrease in pinging force. The results showed that reduction in wire diameters increases the efficiency; however, the effect of increasing the distance between the wires was smaller on the efficiency reduction compared to the increase in wire diameter. The added hydrophobic layer to the mesh collector, increased the efficiency by $62 \%$.
However, only $29 \%$ increase in efficiency was observed when the hydrophobic layer was added to the W0.5-2 collector. Applying the electric field had the most effect on increasing the efficiency (53 times) through changes in the aerodynamic of the system. The charged droplets are captured by the wires working as an electrode and the efficiency reached $82 \%$. The collector shape (wires vs mesh) and the hydrophobic layer were found to increase the efficiency only by $19 \%$ and $3 \%$, in this case. Increasing the wire diameter was found to have negligible effect on the efficiency, while increasing the distance between the wires reduced the efficiency at lower voltages. This was, however compensated at higher voltages which resulted in stronger electric field and more collection of the fog droplets. The maximum SC of 0.55 for the wires without the electric field was increased to 1 in this case. Reaching the same harvesting efficiency with lesser amount of wires used $(S C=1)$, can be significantly beneficial for the cost of the unit. The use of an electric field was found to decrease the pinning force acting on the droplets by disturbing the contact line of the droplets sliding down the wires and reducing the $\mathrm{CAH}$ by three-fold. This allowed for the reduction in the volume of droplets rolling off the wires and opening sites for the nucleation and growth of new droplets. The energy analysis of the system showed that energy consumption was about 100 times lower than the current atmospheric water generators which makes this technique very promising to be used for fog harvesting.

Acknowledgements The authors would like to thank Dr. Seyed Mehdi Borghei and the Department of Chemical and Petroleum Engineering, Sharif University of Technology for supporting this study.

Funding Funding was provided by Iran's National Elites Foundation.

\section{Declarations}

Conflict of interest The authors declare that they have no competing interests

Open Access This article is licensed under a Creative Commons Attribution 4.0 International License, which permits use, sharing, adaptation, distribution and reproduction in any medium or format, as long as you give appropriate credit to the original author(s) and the source, provide a link to the Creative Commons licence, and indicate if changes were made. The images or other third party material in this article are included in the article's Creative Commons licence, unless indicated otherwise in a credit line to the material. If material is not included in the article's Creative Commons licence and your intended use is not permitted by statutory regulation or exceeds the permitted use, you will need to obtain permission directly from the copyright holder. To view a copy of this licence, visit http://creativecommons. org/licenses/by/4.0/. 


\section{References}

1. Fessehaye $\mathrm{M}$ et al (2014) Fog-water collection for community use. Renew Sustain Energy Rev 29:52-62

2. Domen JK et al (2014) Fog water as an alternative and sustainable water resource. Clean Technol Environ Policy 16(2):235-249

3. Zhang $S$ et al (2017) Bioinspired special wettability surfaces: from fundamental research to water harvesting applications. Small 13(3):1602992

4. Zhu H, Guo Z, Liu W (2016) Biomimetic water-collecting materials inspired by nature. Chem Commun 52(20):3863-3879

5. Schemenauer RS, Cereceda P (1994) A proposed standard fog collector for use in high-elevation regions. J Appl Meteorol 33(11):1313-1322

6. Klemm O et al (2012) Fog as a fresh-water resource: overview and perspectives. Ambio 41(3):221-234

7. Qadir M et al (2018) Fog water collection: challenges beyond technology. Water 10(4):372

8. Park K-C et al (2013) Optimal design of permeable fiber network structures for fog harvesting. Langmuir 29(43):13269-13277

9. Azad M et al (2015) Fog collecting biomimetic surfaces: Influence of microstructure and wettability. Bioinspiration Biomim 10(1):016004

10. Ghosh R, Ray TK, Ganguly R (2015) Cooling tower fog harvesting in power plants-a pilot study. Energy 89:1018-1028

11. Ghosh R, Ganguly R (2018) Harvesting water from natural and industrial fogs - opportunities and challenges. In: Basu S, Agarwal AK, Mukhopadhyay A, Patel C (eds) Droplet and spray transport: paradigms and applications. Springer, Berlin, pp 237-266

12. Su Y et al (2019) Smart stretchable Janus membranes with tunable collection rate for fog harvesting. Adv Mater Interfaces 6(22):1901465

13. Feng $R$ et al (2020) A bioinspired slippery surface with stable lubricant impregnation for efficient water harvesting. ACS Appl Mater Interfaces 12:12373-12381

14. Sharma $\mathrm{M}$ et al (2019) Mobility of aqueous and binary mixture drops on lubricating fluid-coated slippery surfaces. Langmuir 35(24):7672-7679

15. Shi W et al (2018) Fog harvesting with harps. ACS Appl Mater Interfaces 10(14):11979-11986

16. Damak M, Varanasi KK (2018) Electrostatically driven fog collection using space charge injection. Sci Adv 4(6):eaao5323
17. Wang L, McCarthy TJ (2016) Covalently attached liquids: instant omniphobic surfaces with unprecedented repellency. Angew Chem Int Ed 55(1):244-248

18. Schemenauer RS, Joe PI (1989) The collection efficiency of a massive fog collector. Atmos Res 24(1-4):53-69

19. de Dios Rivera J (2011) Aerodynamic collection efficiency of fog water collectors. Atmos Res 102(3):335-342

20. Kawasaki K (1960) Study of wettability of polymers by sliding of water drop. J Colloid Sci 15(5):402-407

21. Furmidge $C$ (1962) Studies at phase interfaces. I. The sliding of liquid drops on solid surfaces and a theory for spray retention. J Colloid Sci 17(4):309-324

22. Eral HB et al (2011) Drops on functional fibers: from barrels to clamshells and back. Soft Matter 7(11):5138-5143

23. Parker K (2003) Electrical operation of electrostatic precipitators. IET, London

24. Uchiyama H, Jyumonji M (1995) Field experiments of an electrostatic fog-liquefier. J Electrostat 35(1):133-143

25. Migliavacca G et al (2014) Reduction of PM emissions from biomass combustion appliances: evaluation of efficiency of electrostatic precipitators. Chem Eng Trans 37:25-30

26. Nouri $\mathrm{H}$ et al (2012) Effect of relative humidity on current-voltage characteristics of an electrostatic precipitator. J Electrostat 70(1):20-24

27. Arif $S$ et al (2016) CFD modeling of particle charging and collection in electrostatic precipitators. J Electrostat 84:10-22

28. Chow RT-P (1983) On the ability of drops or bubbles to stick to non-horizontal surfaces of solids. J Fluid Mech 137:1-29

29. Rui $Z$ et al (2015) Contact angle hysteresis in electrowetting on dielectric. Chin Phys B 24(8):086801

30. Gupta R et al (2011) Impact of pinning of the triple contact line on electrowetting performance. Langmuir 27(24):14923-14929

31. Bernetski KA et al (2018) Characterization of electrowetting, contact angle hysteresis, and adhesion on digital microfluidic devices with inkjet-printed electrodes. Microfluid Nanofluid 22(9):1-10

Publisher's Note Springer Nature remains neutral with regard to jurisdictional claims in published maps and institutional affiliations. 